\title{
Passive Cavitation Detection with a Needle Hydrophone Array
}

\author{
Zheng Jiang, Krit Sujarittam, Betul Ilbilgi Yildiz, Robert J. Dickinson, James J. Choi
}

\begin{abstract}
Therapeutic ultrasound and microbubble technologies seek to drive systemically administered microbubbles into oscillations that safely manipulate tissue or release drugs. Such procedures often detect the unique acoustic emissions from microbubbles with the intention of using this feedback to control the microbubble activity. However, most sensor systems reported introduce distortions to the acoustic signal. Acoustic shockwaves, a key emission from microbubbles, are largely absent in reported recording, possibly due to the sensors being too large or too narrowband, or having strong phase distortions. Here, we built a sensor array that countered such limitations with small, broadband sensors and a low phase distorting material. We built 8 needle hydrophones with polyvinylidene fluoride (PVDF, diameter: $2 \mathrm{~mm}$ ) then fit them into a 3D-printed scaffold in a twolayered, staggered arrangement. Using this array, we monitored microbubbles exposed to therapeutically-relevant ultrasound pulses (center frequency: $0.5 \mathrm{MHz}$, peak-rarefactional pressure: 130-597 kPa, pulse length: 4 cycles). Our tests revealed that the hydrophones were broadband with the best having a sensitivity of $-224.8 \pm 3.2 \mathrm{~dB}$ re $1 \mathrm{~V} / \mu \mathrm{Pa}$ from 1 to $15 \mathrm{MHz}$. The array was able to capture shockwaves generated by microbubbles. The signal-tonoise (SNR) ratio of the array was approximately 2 times higher than individual hydrophones. Also, the array could localize microbubbles (-3dB lateral resolution: $2.37 \mathrm{~mm}$ ) and determine the cavitation threshold (between $161 \mathrm{kPa}$ and $254 \mathrm{kPa}$ ). Thus, the array accurately monitored and localized microbubble activities, and may be an important technological step towards better feedback control methods and safer and more effective treatments.
\end{abstract}

Index Terms - Needle hydrophone array, passive cavitation detection, shockwave, cavitation threshold.

\section{INTRODUCTION}

$\mathrm{T}$ echnologies using focused ultrasound and microbubbles have the unique ability to non-invasively and locally treat tissue in deep regions of the body. In these methods, microbubbles are administered intravenously so that they circulate in the bloodstream. The microbubbles are typically composed of a lipid-shell and a heavy gas core and with a diameter of 1 to $10 \mu \mathrm{m}$ [1]. Pulses of focused ultrasound are then applied to the targeted tissue region, causing acoustic cavitation - or acoustically-stimulated bubble activity. During cavitation, the microbubble expands and contracts in response to the rarefactional and compressional phases of the ultrasound

Manuscript received July 06, 2021. This work was supported by Alzheimer's Research UK under grants ARUK-IRG2017A-7. (Corresponding author: James J. Choi.)

Zheng Jiang, Krit Sujarittam, Betul Ilbilgi Yildiz, Robert J. Dickinson, and James J. Choi are with the Department of Bioengineering, Imperial College wave. Depending on the ultrasound pulse shape, the microbubble size and composition, and the surrounding microenvironment, very different kinds of cavitation can be generated $[2,3]$, some of which are therapeutically beneficial, while others are harmful. Since not all of these factors can be accounted for in vivo or in human patients, monitoring the cavitation is considered essential to ensure that the treatment is both safe and effective.

One of the best ways of monitoring the microbubble activity is to detect the unique acoustic emissions that they generate. Using a passive cavitation detector (PCD), microbubble activity can be monitored both non-invasively and in real-time [4-9]. When combined with an array of detectors and beamforming algorithms (e.g., passive acoustic mapping), such microbubble activities can even be localized [10-16]. However, PCDs could benefit from several improvements, such as sensors designed to better decipher the unique sounds that cavitation generates and localize the cavitation event. Previous implementations of PCDs in applications, such as delivering drugs across the bloodbrain barrier (BBB), have mostly used focused polyvinylidene fluoride (PVDF) sensors or lead zirconate titinate (PZT)/PVDF arrays. The former detects the broadband emissions from microbubbles, but they are unable to localize cavitation. Also, due to their large size, focused transducers spatially average the radiated sound on its surface when the acoustic source is outside the focal zone, resulting in less sensitivity and distortion. Linear and hemispherical arrays can localize bubbles [10-15], but they are not ideal for detecting broadband microbubble emissions.

A PCD should be designed to capture the specific acoustic signal that microbubbles generate during therapeutic ultrasound exposure without significant distortions. A microbubble emits acoustic waveforms that are dependent on its oscillatory behavior [3, 6, 17]. At low acoustic pressures, a microbubble produces acoustic emissions near the driving frequency. At higher acoustic pressures, such as those used in therapy, a microbubble will oscillate more nonlinearly and with greater strength, producing stronger acoustic emissions that contain low-order harmonics to the driving pulse. Above a pressure threshold, microbubbles will expand uncontrollably and then violently collapse due to the inertia of the surrounding fluid in a process known as inertial cavitation, which emits an acoustic shockwave $[2,18]$. This waveform contains not only the low

London, London, SW7 2AZ, UK. (e-mail: zheng.jiang18@imperial.ac.uk; krit.sujarittam17@imperial.ac.uk; b.ilbilgi-yildiz18@imperial.ac.uk robert.dickinson@imperial.ac.uk; j.choi@imperial.ac.uk). 
driving frequency, but also high frequency content up to tens of $\mathrm{MHz}$ that appears as a compressional spike in the time domain. Since inertial cavitation has been identified as therapeutically beneficial in some instances and harmful in other instances, it seems prudent that these shockwaves are monitored for their strength, duration, and distribution. However, many of the PCD traces reported in the literature lack the signature shock waveform in the time. This absence of time-domain features can be caused by one or more reasons. First, the sensors are too large. As the sensor's aperture size increases, its ability to detect higher frequency sound becomes increasingly directional due to surface averaging. Second, the sensors or signal conditioning components are too narrowband to capture the acoustic signal in a sufficient frequency range. Third, the sensor or signal conditioning components introduce phase distortions, which can prevent the shock waveform from being captured. Phase distortions are dependent on the overall hydrophone design [19, 20].

Commercial hydrophones are normally used to receive acoustic signals in biomedical applications [21]. They include needle hydrophones $[22,23]$, membrane hydrophones [24, 25], and fiber-optic hydrophones [26, 27]. Among them, needle hydrophones are the most commonly used in lower-intensity therapeutic ultrasound applications. Most needle hydrophones use PVDF as the active element, not only because of its broadband frequency response, its acoustic impedance close to water, and its good receiving sensitivity [28-30], but also because of its accessibility and affordability. When placed close to a single microbubble, they have been proven to capture the unique shock waveforms that bubble collapse generates $[18,31$, 32]. More recently, a study investigated the use three hydrophones to localize cavitation activity [33]. However, the study was not concerned with time-domain signals and the minimization of signal distortions.

Here, we propose a needle hydrophone array for monitoring acoustic cavitation generated at therapeutically-relevant acoustic pressures. The array was composed of 8 individuallyfunctional needle hydrophones which could be spatially distributed in different arrangements by inserting them into other structures, such as 3D-printed scaffolds. Thus, the same 8 needle hydrophones could be re-used and re-distributed in another scaffold to produce a different needle hydrophone array. Each needle hydrophone was able to capture the acoustic emissions from the microbubble while the array configuration sought to boost the signal-to-noise (SNR) ratio and enable imaging.

\section{MATERIALS AND METHODS}

We first designed a needle hydrophone that had a small active element, was broadband, and could be incorporated into a larger array. Each hydrophone was then characterized according to its frequency response. We then assembled 8 hydrophones into a 2-layer staggered arrangement, forming a needle hydrophone array. We evaluated the array's ability to improve the SNR for detecting acoustic shock waves, to localize cavitation activities, and to determine the cavitation threshold.

\section{A. Needle Hydrophone System}

1) Needle hydrophone

Unlike commercial needle hydrophones that have their needle sensors entirely covered in a metal structure, our needle sensor had a coaxial cable extending out of its back, making it flexible enough to fit small spaces. This was an important consideration when scaling up to an array. The needle was $10 \mathrm{~cm}$ long with the sensor and its associated components covering the top 15 $\mathrm{mm}$. The needle sensor had 6 components, which were an active element (PVDF), a backing material (metal rod), an insulating layer, a metal casing, a coaxial cable, and a micro coaxial (MCX) connector. For the active element, we used a 2-mm-in-diameter PVDF disc (Precision Acoustics, Dorchester, UK) with metalized electrodes and poling across the entire surface. A PVDF thickness of 28 was chosen to have an operating frequency range that included 1 to $15 \mathrm{MHz}$. The PVDF was mounted on a metal rod (brass, $2 \mathrm{~mm}$ in diameter, acoustic impedance: 38 MRayls) using a conductive epoxy. The coaxial cable was connected to the metal rod and MCX connector. The metal rod was fixed inside a metal casing (outer diameter: 2.95 $\mathrm{mm}$, inner diameter: $2.3 \mathrm{~mm}$, stainless steel, Tomlinson Tube \& Instrument Ltd, Alcester, UK) with an insulating layer placed in between (Fig. 1). The metal rod was acting as both a backing material and a transmission line for the PVDF.

The needle sensor was assembled in two parts: the needle structure and the PVDF element. The needle structure included the structural support and the wiring for the PVDF element. The MCX connector was first connected to one end of a coaxial cable, while the other end of the cable was soldered to a metal rod. The metal rod was then coated with the insulating layer and fixed inside the metal casing with non-conductive epoxy (3421, Loctite, Düsseldorf, Germany) to form the needle structure. The tip of the needle was then sanded to ensure a flat and smooth surface. The PVDF element was then mounted on the tip of the needle with conductive epoxy (8331, M.G. Chemicals Ltd., Manchester, UK). A thin layer of non-conductive epoxy was



Fig. 1. Cross-sectional image of the flexible-end needle hydrophone (not proportional). The PVDF received acoustic emissions from the microbubbles. In order to extract the electrical signal produced by the sound on the PVDF, we wired the front and back of the PVDF surface. The back was electrically connected through a conductive epoxy, metal rod, and solder, which ultimately fed to a coaxial cable and MCX connector. The front of the PVDF was connected to silver paint, and the metal casing, which ultimately fed to the coaxial cable and MCX connector. These two ends were separated from each other using non-conductive epoxy and insulating layers. The entire needle structure was rigid, but the cables were flexible so that the hydrophone could be inserted into small structures. 
applied around the PVDF to ensure it remained tightly fixed on the needle structure and to avoid a short circuit. Finally, silver paint was used to connect the outer surface of the PVDF element to the metal casing. The metal casing was earthed by the coaxial cable, hence the entire sensor was subsequently earthed to provide effective electrical shielding.

\section{2) Preamplifier}

A preamplifier was built with an OPA659 (Texas Instruments, Texas, USA) operational amplifier [Fig. 2(a)], similar to a design that we built previously [34]. Its frequency response was characterized using a sinusoidal input from a function generator (33500B series, Agilent Technologies Inc., Wood Dale, IL, USA) and recording the output with an oscilloscope (3 series, Tektronix, Oregon, USA). The gain of the preamplifier was constant at 4.7 between 1 and $14 \mathrm{MHz}$ and dropped to 4.6 at 15 $\mathrm{MHz}$ [Fig. 2(b)].
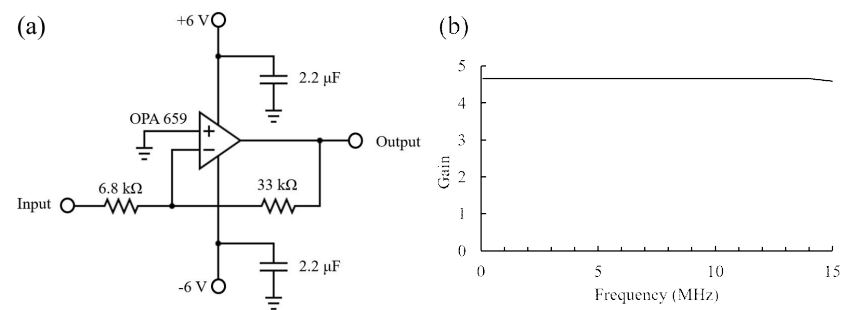

Fig. 2. Preamplifier of the needle hydrophone. (a) Circuit diagram. (b) Frequency response of the preamplifier.

\section{3) Frequency Response}

The end-of-cable receiving sensitivity of each needle hydrophone was calibrated in a water bath to a commercial needle hydrophone (diameter: $2 \mathrm{~mm}$; Precision Acoustics, Dorchester, UK) [24, 35], which we will refer to as the PA hydrophone. The absolute receiving sensitivity (in $\mathrm{mV} / \mathrm{MPa}$ ) of this hydrophone was calibrated by Precision Acoustics. An inhouse built PVDF piston emitter with an active diameter of 10 $\mathrm{mm}$ and a thickness of $110 \mu \mathrm{m}$ was used to generate ultrasound pulses between 1 and $15 \mathrm{MHz}$ in steps of $1 \mathrm{MHz}$. The PA hydrophone was placed $25 \mathrm{~cm}$ away from the surface of the PVDF emitter to ensure that it was always in the far field (Fig. 3). The lateral maximum was found by moving the PA hydrophone until the strongest acoustic signal was found. The PVDF emitter was excited by a function generator (33500B series, Agilent Technologies Inc., Wood Dale, IL, USA) through a power amplifier $(2100 \mathrm{~L}$, Electronics \& Innovation, Ltd., Rochester, NY, USA). Short tone bursts were generated by the PVDF emitter and received by the PA hydrophone. Received signals were digitized by a GaGe data acquisition system (Oscar 16, Dynamic Signals, San Bruno, CA, USA) and recorded on a computer.

Received signals were first windowed to remove reflections and electrical interference. The hydrophone voltage readings were then recorded after confirming that the pulse contained the intended centre frequency. Our needle hydrophone was placed at the same location and its absolute receiving value was recorded with the same method. As both the PA hydrophone and our hydrophone had an active diameter of $2 \mathrm{~mm}$, no further correction on spatial averaging was needed. The absolute receiving sensitivity level $S_{n}$ (in decibels relative to $1 \mathrm{~V} / \mu \mathrm{Pa}$ )

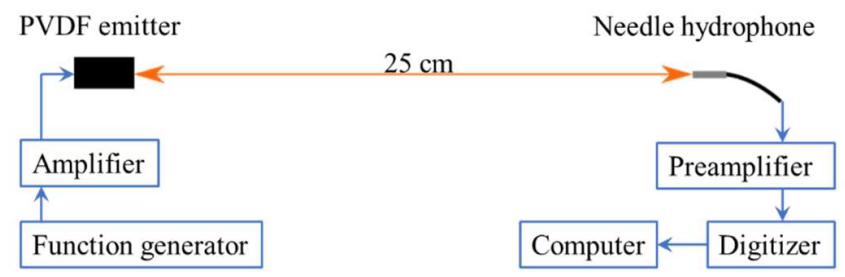

Fig. 3. Experimental setup used to characterize the frequency response of the needle hydrophone. A PVDF emitter with an active diameter of $10 \mathrm{~mm}$ was used to emit ultrasound pulses between 1 and $15 \mathrm{MHz}$ in steps of $1 \mathrm{MHz}$. Either our needle hydrophone or a commercial needle hydrophone was placed $25 \mathrm{~cm}$ away from the emitter to ensure that it was in the far field for all of the frequencies tested.

of our needle hydrophone was then calculated as:

$$
S_{n}=20 \times \log \left(\frac{V_{n}(f)}{V_{c}(f)} \times S_{c} \times 10^{9}\right)
$$

where $S_{c}$ is the sensitivity of the commercial needle hydrophone (in $\mathrm{mV} / \mathrm{MPa}$ ), $V_{c}(f)$ and $V_{n}(f)$ are the received absolute value (in $\mathrm{mV}$ ) from the PA hydrophone and our needle hydrophone, respectively. We used the same method to calibrate all 8 of our needle hydrophones.

\section{B. Needle Hydrophone Array \\ 1) Design and Construction}

Eight needle hydrophones were assembled into a 3D-printed frame to form an array. The array was formed by two layers of the needle hydrophones systems with a staggered arrangement [Fig. 4]. This arrangement was adapted to minimize the pitch size while keeping a reasonably large aperture. The element-toelement spacing was $2.96 \mathrm{~mm}$, but the effective projected pitch size to the imaging plane was $1.48 \mathrm{~mm}$ due to the staggered arrangement. The active aperture size were $12.63 \mathrm{~mm}$ with a width of $4.56 \mathrm{~mm}$. The imaging plane was parallel to the array and to the needles' axes. The array (relative locations of elements) were determined with the acoustic time-of-fight method.



Fig. 4. The arrangement of the 8-element needle hydrophone array.

\section{2) Experimental Setup}

The experiments were performed in a tank filled with degassed and deionized water [Fig. 5(a))]. A focused 0.5-MHz transducer (Sonic Concepts Inc, Bothell, WA, USA) was used to sonicate microbubbles. Its focal length was $64 \mathrm{~mm}$, and its focal spot had an axial and a lateral full-width at half-maximum of pressure field (FWHM) of $4 \mathrm{~mm}$ and $37 \mathrm{~mm}$, respectively. Water-diluted microbubbles were flowing inside of a $0.63-\mathrm{mm}$ wall-less channel, which was formed within a block of hydrogel. The channel was overlapping with the focal spot and aligned perpendicularly to the propagation direction of ultrasound waves. The array was used to receive microbubble emissions. It was aligned directly facing the sonicated region of the channel at a distance of $40 \mathrm{~mm}$, and with its long axis parallel to the ultrasound wave propagation direction. 
The wall-less channel used in the experiments was within a soft polyacrylamide hydrogel (modulus of elasticity of $8.44 \pm$ $0.82 \mathrm{kPa})[34,36]$, so that the reflection from the gel-water interface could be minimized. Lipid-shelled microbubbles with a heavy gas core were made in-house (average radius of $0.66 \pm$ $0.38 \mu \mathrm{m}$ ) using a previously described method [37]. The microbubbles were diluted with degassed and deionized water. During the experiments, the microbubble suspension was constantly stirred by a magnetic stirrer and drawn through the channel by a syringe pump (Harvard Apparatus, Holliston, MA, USA). The pulling rate was $0.2 \mathrm{~mL} / \mathrm{min}$ to ensure replenishment of microbubbles between each sonication.

The $0.5-\mathrm{MHz}$ focused transducer was excited by a function generator (33500B series, Agilent Technologies Inc., Wood Dale, IL, USA) through a power amplifier (2100L, Electronics \& Innovation, Ltd., Rochester, NY, USA). The $0.5 \mathrm{MHz}$ focused transducer was excited with a short pulse (pulse length: 4 cycles, phase: 235 degree) and generated a 5-cycle waveform. Its $1^{\text {st }}$ and $5^{\text {th }}$ negative peaks approximately had approximately half the amplitude of the center 3 cycles [Fig. 5(b)]. The microbubble emissions were captured by all 8 needle hydrophones in the array and received by an 8-channel receiver (AFE5808AEVM, Texas Instruments, Texas, USA). The signals were digitized by a data capture card (digitizing rate: 40MHz, TSW1400EVM, Texas Instruments, Texas, USA) before being stored on a computer.

\section{Capturing Shockwaves Generated by a Single Microbubble}

Microbubbles were diluted so that only a few bubbles would be in the focus of the transducer during each sonication, allowing us to confirm whether shockwaves from single bubbles could be captured. The microbubbles were diluted with water at a ratio of $1: 8,000,000$. The $0.5 \mathrm{MHz}$ focused transducer was excited to transmit ultrasound with a maximum peaknegative pressure (PNP) of $0.43 \mathrm{MPa}$. Twenty control sonications were first performed where only water was flowing in the channel while sonicating. Microbubble solution was then flowed through the channel and hundreds of sonications were performed.

The signals received by the array were processed to find shockwaves captured. The background signal was obtained by averaging the control signals, which was then subtracted from the microbubble-solution signals. The background-subtracted signals containing shockwaves were selected by an automatic Matlab algorithm. Since we could identify when sonication of a dilute microbubble solution produced only a single shockwave, we used this event to determine the time of flight between the bubble and each sensor. The averaged array signal was then obtained by time delaying the 8 shockwave signals and then averaging across all 8 channels. The averaged shockwave signal was analyzed in both the time and frequency domains. The shockwaves were then windowed and the SNR was calculated.

\section{Monitoring a Microbubble Cloud}

In this experiment, the microbubbles were diluted to a ratio

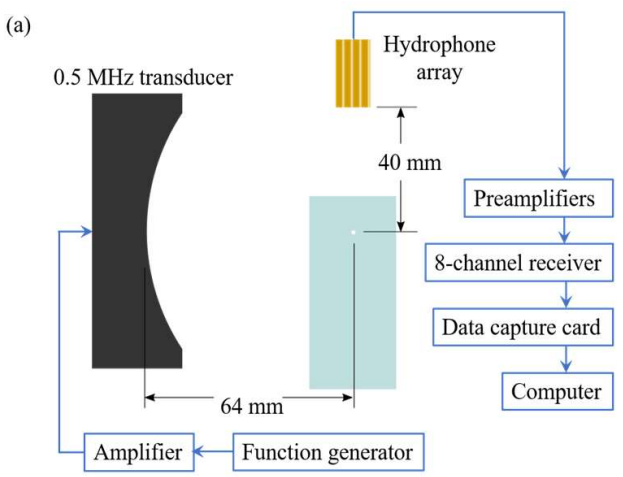

(b)

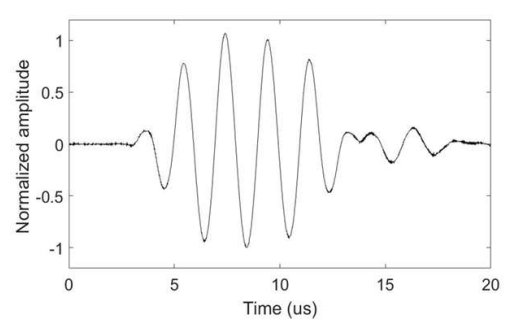

Fig. 5. Experimental setup using the needle hydrophone array to capture microbubble emissions. (a) Microbubbles flowing inside a $0.63-\mathrm{mm}$-indiameter wall-less channel were sonicated by a $0.5 \mathrm{MHz}$ focused transducer. An 8-element needle hydrophone array captured the radiated microbubble emissions. (b) The $0.5-\mathrm{MHz}$ transducer generated a waveform with 5 negative peaks, with the $1^{\text {st }}$ and $5^{\text {th }}$ peaks having an amplitude approximately $50 \%$ smaller than that of the other 3 negative peaks.

of $1: 1,000$ to ensure that many microbubbles were present within the sonication region. The $0.5 \mathrm{MHz}$ transducer was excited to generate the same waveform [Fig. 5 (b)]. The transmitted PNP increased from $130 \mathrm{kPa}$ to $597 \mathrm{kPa}$ in steps of $31 \mathrm{kPa}$. Twenty sonications were run for each PNP.

The received microbubble signals were high-pass filtered (1.25 MHz cut-off frequency, Hamming window) to remove reflections and reverberation of the excitation pulse before imaging. We used an axial temporal position passive acoustic mapping (ATP-PAM) algorithm to localize the microbubble cloud. This algorithm combines time-of-flight calculations and delay-and-sum beamforming to improve the imaging axial resolution and to remove artifacts $[10,38]$. The time window used was $6 \mu$ s and the sound speed in water was assumed to be $1498 \mathrm{~m} / \mathrm{s}$. We then visualized the time-frequency representation of the delayed and summed signal at the brightest spot in the reconstructed image using the Stockwell transform (Stransform) [39]. This transform allows visualization of the frequency components of a signal with a temporal resolution that improves with increasing frequency. Finally, the cavitation threshold was determined by analyzing broadband components within the received microbubble signals. Fast fourier transform (FFT) was performed on all filtered signals (high-pass filtered at $2 \mathrm{MHz}$ ). The frequency spectrums were then sub-divided using Tukey windows (stop band $=0.25 \mathrm{MHz}$, cosine fraction $=0.8$ ) to remove all harmonic frequencies so that only broadband components were left. The broadband energy was taken as the sum of squared values of the spectrum.

\section{RESULTS}

\section{A. Frequency Response}

Our needle hydrophone system, which included the needle 
and the pre-amplifier, had a fairly flat frequency response between 1 and $15 \mathrm{MHz}$, similar to the PA hydrophone [Fig. 6(a)]. One of our best needle hydrophones had a sensitivity variation of less than $10 \mathrm{~dB}$ over the frequency range, similar to the PA hydrophone. However the PA hydrophone had a smoother response at lower frequencies with a more marked drop-off near $15 \mathrm{MHz}$. The sensitivity level of our needle hydrophone system was also about $30 \mathrm{~dB}$ higher than that of the PA hydrophone.

All 8 needle hydrophone systems used in the array were calibrated and some variation between them were found [Fig. 6(b)]. The difference in sensitivity level between the 8 hydrophones was smaller at the lower frequencies (e.g., $6 \mathrm{~dB}$ at $1 \mathrm{MHz})$, but increased at the higher frequencies $(26 \mathrm{~dB}$ at 15 $\mathrm{MHz}$ ). All 8 hydrophones were able to receive signals up to 15 $\mathrm{MHz}$, though the consistency in frequency response varied.
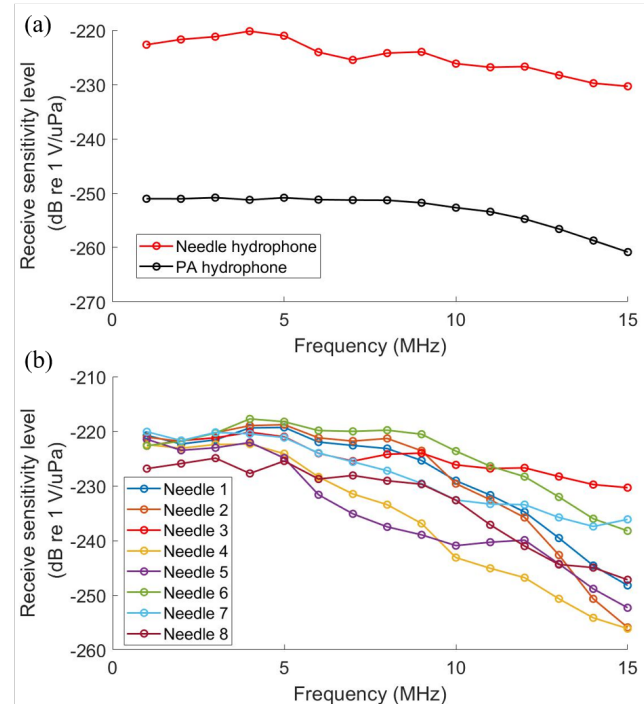

Fig. 6. End-of-cable frequency response of the needle hydrophones. The frequency response of a 2-mm-in-diameter needle hydrophone (Needle 3) was (a) compared to a Precision Acoustics (PA) hydrophone and (b) all hydrophones of the 8-needle array.

\section{B. Capturing Acoustic Shockwaves}

All 8 needle hydrophones in the array were able to capture the shock waveform generated by single microbubbles exposed to a 4-cycle, 0.5-MHz ultrasonic pulse at $0.43 \mathrm{MPa}$ [Fig. 7(a)]. In signals where one shockwave per period of the excitation pulse was present, three shockwaves could be observed in each channel, which was likely due to the 3 large rarefactional phases of the pulse. The amplitudes of the shockwaves varied between channels. The range was 200 to 480 (arbitrary units a.u.). On average, the variations were $350.6 \pm 90.1$ (standard deviation) across all channels. As the signals captured by all 8 hydrophones had the characteristic shockwave shape and had similar waveforms, we were confident that the shockwaves were generated by a single microbubble. If acoustic signals contained more than one compressional spike in a single driving signal cycle, they were considered to have multiple microbubbles and were discarded.

An average shock waveform was obtained from the 8 signals after temporal shift and alignment, which resulted in 3 clear shockwaves [Fig. 7(b)]. The mean of the SNR of the shockwave signals captured by individual hydrophones was $10 \mathrm{~dB} \pm 1.6 \mathrm{~dB}$ (SD). While the SNR of the averaged signal was $19.8 \mathrm{~dB}$, about 2 times higher. The rise time of a shockwave in the averaged signal from $10 \%$ to $90 \%$ of the peak was $0.12 \pm 0.02 \mu \mathrm{s}$. The standard deviations are based on the three shockwaves produced from a single pulse. Harmonic (i.e. $1 \mathrm{MHz}, 1.5 \mathrm{MHz}$ ) and ultra-harmonic (i.e. $0.75 \mathrm{MHz}, 1.25 \mathrm{MHz}$ ) frequencies were observed in the frequency domain [Fig. 7(c)].

(a)
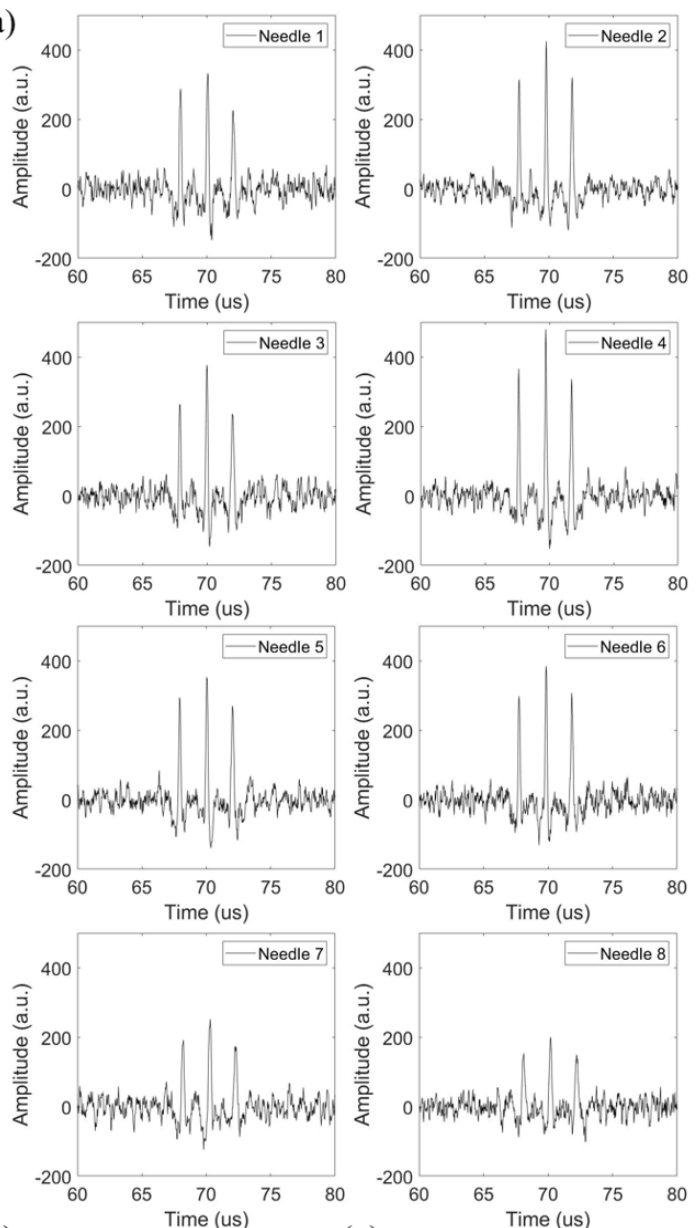

(b)
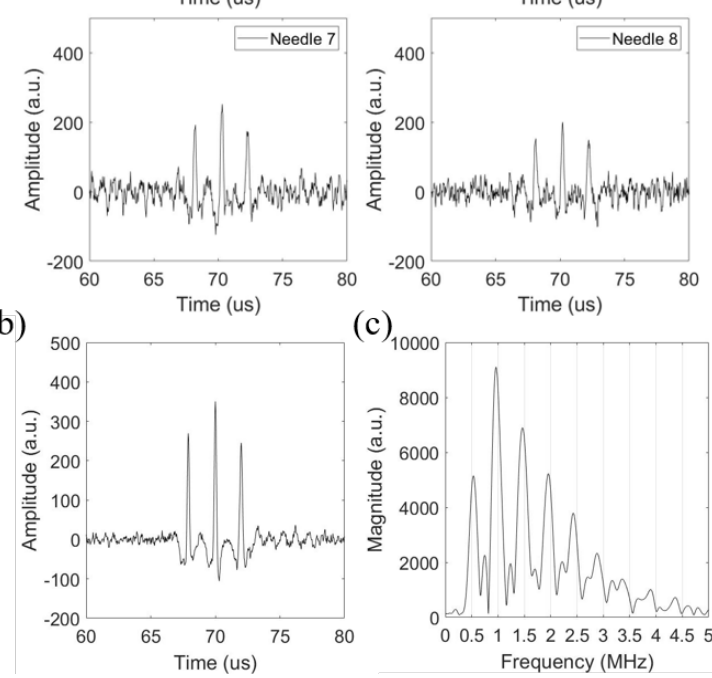

Fig. 7. Shockwaves captured by the 8-element hydrophone array. Microbubbles were exposed to an ultrasound field $(0.5 \mathrm{MHz}, 0.43 \mathrm{MPa})$. (a) Shockwaves generated by a single microbubble were captured by individual hydrophones. The signals were then averaged after realignment and shown in the (b) time domain and (c) frequency domain.

\section{Monitoring Microbubble Activity}

The hydrophone array was able to localize the microbubbles using the ATP-PAM algorithm [Fig. 8 (a)]. The $-3 \mathrm{~dB}$ axial and lateral width of the spot were $6.15 \mathrm{~mm}$ and $3.00 \mathrm{~mm}$, respectively, while the diameter of the microbubble-filled channel was $0.63 \mathrm{~mm}$. The spatial resolution was limited by the 
small active aperture size of the array $(12.63 \mathrm{~mm})$ and the 4 cycle pulse length. Increasing the aperture size by adding more elements and shortening the pulse length would improve the axial and lateral resolution. However, increasing the aperture size by increasing the array spacing would not be a good approach as this would increase the pitch size, which was already large for the frequency used to image (above $1.25 \mathrm{MHz}$ ), as seen by the diagonal grating-lobe artefacts.

The hydrophone array was able to detect broadband microbubble emissions. Here, the S-transform was used to analyze the microbubble activity, because it provides a better characterization of the physical nature of the cavitation activity rather than focusing on the periodic nature of the spikes. When the microbubbles were exposed to $161 \mathrm{kPa}$, only low order harmonics were detected from the array [Fig. 8(b)]. As the applied pressure increased, higher harmonics were observed until $254 \mathrm{kPa}$ [Fig. 8(c)], where broadband signals were then detected. At $597 \mathrm{kPa}$, very clear broadband signals could be detected [Fig. 8(d)]. Based on these recordings, the cavitation threshold lay between $161 \mathrm{kPa}$ and $254 \mathrm{kPa}$. The energy of the broadband signals for the 20 trials at each PNP revealed that energy of acoustic emissions increased with pressure as expected [Fig. 8(e)].


(e)

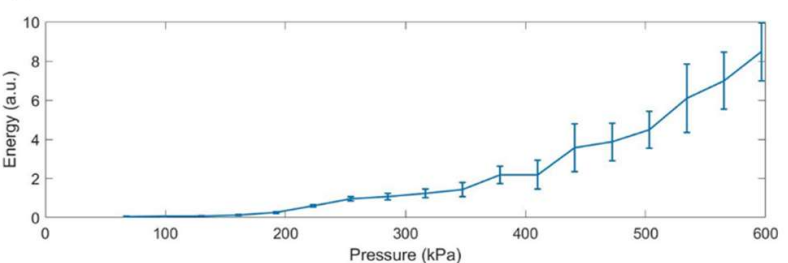

Fig. 8. Passive cavitation detection with the needle hydrophone array. The microbubbles were exposed in an ultrasound field (frequency: $0.5 \mathrm{MHz}$, cycles: 4, phase: 235 degree, PNP: $130 \mathrm{kPa}$ to $597 \mathrm{kPa}$ at a step of $31 \mathrm{kPa}$ ) and the microbubble emissions were captured by the hydrophone array. The microbubble activity was imaged using (a) ATP-PAM. The S-transform of the brightest pixel in the ATP-PAM images revealed the frequency content over time at (b) $161 \mathrm{kPa}$, (c) $254 \mathrm{kPa}$, (d) and $597 \mathrm{kPa}$. (e) The sum of squared broadband signals of all 20 trials at different PNP was also calculated.

\section{DISCUSSION}

We have shown that our needle hydrophone array was capable of capturing acoustic shockwaves generated from inertial cavitation. It was also able to localize cavitation activity and determine the cavitation threshold.
The frequency response of our needle hydrophone [Fig. 6(a)] was similar to that of the PA hydrophone. However, the difference in frequency response among the 8 needle hydrophones was obvious [Fig. 6(b)]. This may be caused by many factors, such as the heterogeneity within the PVDF elements, the thickness of the conductive epoxy between the PVDF and the metal rod, and variations within the many components used. The dimensions of the PVDF was chosen to provide a flat frequency response between 1 and $15 \mathrm{MHz}$. To achieve this, we sought a design that placed the thickness-mode resonance frequency above this range and a radial resonance frequency below this range. The $28 \mu \mathrm{m}$ thickness gave a working frequency range up to about $40 \mathrm{MHz}$ with a $\lambda / 4$ thickness resonance frequency of approximately $20 \mathrm{MHz}$ [40]. Moreover, the $2 \mathrm{~mm}$ diameter led to a radial resonance frequency of approximately $0.42 \mathrm{MHz}$ [41]. Our needle hydrophones were more sensitive than the PA hydrophone (30 $\mathrm{dB}$ higher in sensitivity for our best one), this may have been due to the PA hydrophone was more heavily damped by the backing material. The heavier damping also explains why the PA hydrophone had a smoother frequency response [Fig. 6(a)].

All 8 hydrophones within the array were able to capture shockwaves generated by a single microbubble [Fig. 7(a)]. Ultrasound pulses having 5 negative peaks were used to sonicate, while only 3 shockwaves were visualized in each captured signal. This difference was most likely due to the microbubble responding most strongly to the 3 large negative peaks [Fig 5(b)]. Although all 8 needle hydrophones showed three clear shockwaves, the amplitude detected from each was obviously different. This was caused not only by the different sensitivities, but also by the different locations [Fig. 5(a)]. As we used a flat array aperture, the hydrophones on the edges faced the microbubble at larger angles. For the same hydrophone, its directional response dropped as the angle of incident between the direction of wave propagation and the sensor surface decreased [42, 43]. So, in addition to the incoming signal being reduced by more attenuation, the furthest hydrophone was also less sensitive than hydrophones directly facing the microbubble. The SNR of the averaged shockwave signal was about 2 times higher than individuals. This can be very useful when the SNR of each hydrophone signal is insufficient. The improvement on SNR was smaller than theoretical predictions of approximately 2.8 . This was likely due to the edge sensors having less sensitivity. The needle hydrophones can receive signals up to $15 \mathrm{MHz}$, but when only a single microbubble producing ultrasound, we were only able to capture signal up to around $4 \mathrm{MHz}$ [Fig. 7(c)]. This was likely because the high-frequency components within the shockwave were more severely attenuated. However, clear harmonics and ultra-harmonics below $5 \mathrm{MHz}$ were captured.

The hydrophone array was capable of monitoring microbubble activities. It was able to localize microbubble activities and capture broadband signals, allowing it to determine the cavitation threshold [Fig. 8]. The resolution of the reconstructed image was approximately what we expected: The $-3 \mathrm{~dB}$ lateral resolution of sound mapped from microbubbles was $2.37 \mathrm{~mm}$, which was obtained by subtracting 
the channel diameter from the localized sound's $-3 \mathrm{~dB}$ width. This value was approximately equal to the theoretical lateral resolution of $2.82 \mathrm{~mm}$, which assumed an f-number of 3.17 and a wavelength of $1 \mathrm{~mm}$ [44]. The axial resolution was dictated by the pulse length of the excitation pulse. The nominal pulse length of $6 \mu$ s was set according to the length of the central three peaks. The axial resolution was approximately the propagation distance the wave traveled in $6 \mu$ s. The resolution was sufficient enough to prove that microbubble activity could be localized with this array. The resolution can be improved by increasing the aperture size, adding more hydrophone elements, and using a focused arrangement $[44,45]$. The array was also able to determine the threshold of cavitation [Fig. 8(e)]. The likelihood of tissue damage in ultrasound-mediated drug delivery increased with increasingly applied peak-rarefactional pressure. Hence to ensure a safe and successful treatment, it is important to use a pressure only slightly above the cavitation threshold which the array will permit.

While the presented needle hydrophone array has been used here in an in vitro setting, we anticipate that a similar array design could be useful in an in vivo and clinical setting. Some concerns with using such an array in biological tissue is that the bubble signal will attenuate and experience nonlinear distortions with increasing penetration depth. One could limit applications to superficial settings which would include most organs of the mouse and rat, and the skin and carotid artery of human patients. To achieve deeper acoustic monitoring, one could increase the number of elements or boost the sensitivity of each sensor by improving the pre-amplifier or converting to other materials, such as PZT. We are encouraged that this may be possible as a single PVDF sensor was proven to detect bubble acoustic emissions across the human skull [9].

\section{CONCLUSION}

Here, we designed a PCD system that can capture the temporal and frequency features of the microbubble's acoustic emissions while also being able to localize cavitation activity. The lack of distortion introduced is best conveyed by its ability to recover the shock waveform that bubbles produce during inertial cavitation. Thus, a small and broadband sensor array design allows key detection features that PCD systems could benefit from. Such designs have the potential to improve feedback systems or provide new information which new feedback control systems can utilize, hopefully leading to targeted, safe, and effective treatments.

\section{ACKNOWLEDGMENT}

The authors would like to thank Paschal Egan, Niraj Kanabar for their assistance with the electronics, and Marta Garcia Bellmunt for her assistance with 3D printing.

\section{REFERENCES}

[1] E. Stride, T. Segers, G. Lajoinie, S. Cherkaoui, T. Bettinger, M. Versluis, et al., "Microbubble agents: New directions," Ultrasound in medicine \& biology, vol. 46, pp. 1326-1343, 2020.

[2] C. C. Church and E. L. Carstensen, "SStable" inertial cavitation," Ultrasound in medicine \& biology, vol. 27, pp. 1435-1437, 2001.
[3] C. Coussios, C. Farny, G. Ter Haar, and R. Roy, "Role of acoustic cavitation in the delivery and monitoring of cancer treatment by highintensity focused ultrasound (HIFU)," International journal of hyperthermia, vol. 23, pp. 105-120, 2007.

[4] M. A. O'Reilly and K. Hynynen, "Blood-brain barrier: Real-time feedback-controlled focused ultrasound disruption by using an acoustic emissions-based controller," Radiology, vol. 263, pp. 96-106, 2012.

[5] A. N. Pouliopoulos, S.-Y. Wu, M. T. Burgess, M. E. Karakatsani, H. A. Kamimura, and E. E. Konofagou, "A Clinical System for Non-invasive Blood-Brain Barrier Opening Using a Neuronavigation-Guided SingleElement Focused Ultrasound Transducer," Ultrasound in medicine \& biology, 2019.

[6] Y.-S. Tung, F. Vlachos, J. J. Choi, T. Deffieux, K. Selert, and E. E. Konofagou, "In vivo transcranial cavitation threshold detection during ultrasound-induced blood-brain barrier opening in mice," Physics in Medicine \& Biology, vol. 55, p. 6141, 2010.

[7] N. McDannold, N. Vykhodtseva, and K. Hynynen, "Targeted disruption of the blood-brain barrier with focused ultrasound: association with cavitation activity," Physics in Medicine \& Biology, vol. 51, p. 793, 2006.

[8] S. V. Morse, A. N. Pouliopoulos, T. G. Chan, M. J. Copping, J. Lin, N. J. Long, et al., "Rapid Short-pulse Ultrasound Delivers Drugs Uniformly across the Murine Blood-Brain Barrier with Negligible Disruption," Radiology, p. 181625, 2019.

[9] M. A. O'Reilly and K. Hynynen, "A PVDF receiver for ultrasound monitoring of transcranial focused ultrasound therapy," IEEE transactions on Biomedical Engineering, vol. 57, pp. 2286-2294, 2010.

[10] H. J. Davies, S. V. Morse, M. J. Copping, K. Sujarittam, V. D. Bourgin, M.-X. Tang, et al., "Imaging With Therapeutic Acoustic Wavelets-Short Pulses Enable Acoustic Localization When Time of Arrival is Combined With Delay and Sum," IEEE Transactions on Ultrasonics, Ferroelectrics, and Frequency Control, vol. 68, pp. 178-190, 2020.

[11] J. J. Choi, R. C. Carlisle, C. Coviello, L. Seymour, and C.-C. Coussios, "Non-invasive and real-time passive acoustic mapping of ultrasoundmediated drug delivery," Physics in Medicine \& Biology, vol. 59, p. 4861, 2014.

[12] C. M. Coviello, R. J. Kozick, A. Hurrell, P. P. Smith, and C.-C. Coussios, "Thin-film sparse boundary array design for passive acoustic mapping during ultrasound therapy," IEEE transactions on ultrasonics, ferroelectrics, and frequency control, vol. 59, pp. 2322-2330, 2012.

[13] J. Collin, C. Coviello, E. Lyka, T. Leslie, and C. C. Coussios, "Real-time three-dimensional passive cavitation detection for clinical high intensity focussed ultrasound systems," in Proceedings of Meetings on Acoustics ICA2013, 2013, p. 075023.

[14] Z. Hu, L. Xu, C.-Y. Chien, Y. Yang, Y. Gong, D. Ye, et al., "Threedimensional Transcranial Microbubble Cavitation Localization by Four Sensors," IEEE Transactions on Ultrasonics, Ferroelectrics, and Frequency Control, 2021.

[15] M. A. O'Reilly, R. M. Jones, and K. Hynynen, "Three-dimensional transcranial ultrasound imaging of microbubble clouds using a sparse hemispherical array," IEEE Trans Biomed Eng, vol. 61, pp. 1285-1294, 2014.

[16] V. A. Salgaonkar, S. Datta, C. K. Holland, and T. D. Mast, "Passive cavitation imaging with ultrasound arrays," The Journal of the Acoustical Society of America, vol. 126, pp. 3071-3083, 2009.

[17] F. Vignon, W. T. Shi, J. E. Powers, E. C. Everbach, J. Liu, S. Gao, et al., "Microbubble cavitation imaging," IEEE transactions on ultrasonics, ferroelectrics, and frequency control, vol. 60, pp. 661-670, 2013.

[18] J. H. Song, K. Johansen, and P. Prentice, "An analysis of the acoustic cavitation noise spectrum: The role of periodic shock waves," The Journal of the Acoustical Society of America, vol. 140, pp. 2494-2505, 2016.

[19] K. A. Wear, P. M. Gammell, S. Maruvada, Y. Liu, and G. R. Harris, "Improved measurement of acoustic output using complex deconvolution of hydrophone sensitivity," IEEE transactions on ultrasonics, ferroelectrics, and frequency control, vol. 61, pp. 62-75, 2014.

[20] K. A. Wear, Y. Liu, P. M. Gammell, S. Maruvada, and G. R. Harris, "Correction for frequency-dependent hydrophone response to nonlinear pressure waves using complex deconvolution and rarefactional filtering: Application with fiber optic hydrophones," IEEE transactions on ultrasonics, ferroelectrics, and frequency control, vol. 62, pp. 152-164, 2015.

[21] G. R. Harris, "Progress in medical ultrasound exposimetry," IEEE transactions on ultrasonics, ferroelectrics, and frequency control, vol. 52, pp. 717-736, 2005 .

[22] P. Lewin, "Miniature piezoelectric polymer ultrasonic hydrophone probes," Ultrasonics, vol. 19, pp. 213-216, 1981. 
[23] M. Platte, "A polyvinylidene fluoride needle hydrophone for ultrasonic applications," Ultrasonics, vol. 23, pp. 113-118, 1985.

[24] R. Preston, D. Bacon, A. Livett, and K. Rajendran, "PVDF membrane hydrophone performance properties and their relevance to the measurement of the acoustic output of medical ultrasonic equipment," Journal of Physics E: Scientific Instruments, vol. 16, p. 786, 1983.

[25] V. Wilkens, S. Sonntag, and O. Georg, "Robust spot-poled membrane hydrophones for measurement of large amplitude pressure waveforms generated by high intensity therapeutic ultrasonic transducers," The Journal of the Acoustical Society of America, vol. 139, pp. 1319-1332, 2016.

[26] V. Bull, J. Civale, I. Rivens, and G. Ter Haar, "A comparison of acoustic cavitation detection thresholds measured with piezo-electric and fiberoptic hydrophone sensors," Ultrasound in medicine \& biology, vol. 39, pp. 2406-2421, 2013

[27] J. E. Parsons, C. A. Cain, and J. B. Fowlkes, "Cost-effective assembly of a basic fiber-optic hydrophone for measurement of high-amplitude therapeutic ultrasound fields," The Journal of the Acoustical Society of America, vol. 119, pp. 1432-1440, 2006.

[28] K. S. Ramadan, D. Sameoto, and S. Evoy, "A review of piezoelectric polymers as functional materials for electromechanical transducers," Smart Materials and Structures, vol. 23, p. 033001, 2014.

[29] K. K. Shung, J. Cannata, and Q. Zhou, "Piezoelectric materials for high frequency medical imaging applications: A review," Journal of Electroceramics, vol. 19, pp. 141-147, 2007.

[30] Q. Zhang and P. A. Lewin, "PVDF polymers: Imaging transducers and ultrasonic hydrophone probes," Ferroelectrics, vol. 171, pp. 381-403, 1995.

[31] K. Johansen, J. H. Song, K. Johnston, and P. Prentice, "Deconvolution of acoustically detected bubble-collapse shock waves," Ultrasonics, vol. 73 pp. 144-153, 2017.

[32] J. H. Song, A. Moldovan, and P. Prentice, "Non-linear acoustic emissions from therapeutically driven contrast agent microbubbles," Ultrasound in medicine \& biology, vol. 45, pp. 2188-2204, 2019.

[33] M. Lafond, N. Asquier, J.-L. A. Mestas, A. Carpentier, S.-i. Umemura, and C. Lafon, "Evaluation of a Three-Hydrophone Method for 2-D Cavitation Localization," IEEE transactions on ultrasonics, ferroelectrics, and frequency control, vol. 65, pp. 1093-1101, 2018.

[34] Z. Jiang, R. J. Dickinson, T. L. Hall, and J. J. Choi, "A PZT-PVDF Stacked Transducer for Short-pulse Ultrasound Therapy and Monitoring," IEEE Transactions on Ultrasonics, Ferroelectrics, and Frequency Control, 2021.

[35] R. A. Smith and D. R. Bacon, "A multiple - frequency hydrophone calibration technique," The Journal of the Acoustical Society of America, vol. 87 , pp. 2231-2243, 1990.

[36] J. R. Tse and A. J. Engler, "Preparation of hydrogel substrates with tunable mechanical properties," Current protocols in cell biology, vol. 47 pp. 10.16. 1-10.16. 16, 2010.

[37] A. N. Pouliopoulos, S. Bonaccorsi, and J. J. Choi, "Exploiting flow to control the in vitro spatiotemporal distribution of microbubble-seeded acoustic cavitation activity in ultrasound therapy," Physics in Medicine \& Biology, vol. 59, p. 6941, 2014.

[38] J. Gateau, J.-F. Aubry, M. Pernot, M. Fink, and M. Tanter, "Combined passive detection and ultrafast active imaging of cavitation events induced by short pulses of high-intensity ultrasound," IEEE transactions on ultrasonics, ferroelectrics, and frequency control, vol. 58, pp. 517-532, 2011.

[39] R. G. Stockwell, L. Mansinha, and R. Lowe, "Localization of the complex spectrum: the S transform," IEEE transactions on signal processing, vol. 44, pp. 998-1001, 1996.

[40] P. C. Beard, A. M. Hurrell, and T. N. Mills, "Characterization of a polymer film optical fiber hydrophone for use in the range 1 to $20 \mathrm{MHz}$ : A comparison with PVDF needle and membrane hydrophones," IEEE Transactions on ultrasonics, ferroelectrics, and frequency control, vol. 47, pp. 256-264, 2000.

[41] K. A. Wear, Y. Liu, and G. R. Harris, "Pressure pulse distortion by needle and fiber-optic hydrophones due to nonuniform sensitivity," IEEE transactions on ultrasonics, ferroelectrics, and frequency control, vol. 65 , pp. 137-148, 2017.

[42] D. G. Shombert, S. W. Smith, and G. R. Harris, "Angular response of miniature ultrasonic hydrophones," Medical physics, vol. 9, pp. 484-492, 1982.

[43] K. A. Wear, C. Baker, and P. Miloro, "Directivity and frequencydependent effective sensitive element size of needle hydrophones:
Predictions from four theoretical forms compared with measurements," IEEE transactions on ultrasonics, ferroelectrics, and frequency control, vol. 65 , pp. 1781-1788, 2018.

[44] M. Gyöngy and C.-C. Coussios, "Passive cavitation mapping for localization and tracking of bubble dynamics," The Journal of the Acoustical Society of America, vol. 128, pp. EL175-EL180, 2010.

[45] R. M. Jones, M. A. O’Reilly, and K. Hynynen, "Transcranial passive acoustic mapping with hemispherical sparse arrays using CT-based skullspecific aberration corrections: a simulation study," Physics in Medicine \& Biology, vol. 58, p. 4981, 2013.

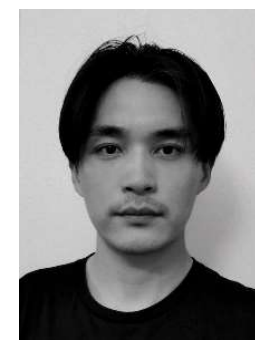

Zheng Jiang received the B.Eng. degree in aircraft airworthiness technology (Aircraft Design and Engineering) from the Nanjing University of Aeronautics and Astronautics, Nanjing, China, in 2015, and the M.Eng. degree in mechanical engineering and theory from the State Key Laboratory of Mechanics and Control of Mechanical Structures, Nanjing University of Aeronautics and Astronautics, in 2018. He is currently pursuing the $\mathrm{Ph} . \mathrm{D}$. degree with the Department of Bioengineering, Imperial College London, London, U.K. His research interests include transducer design, ultrasound simulation, and skull imaging

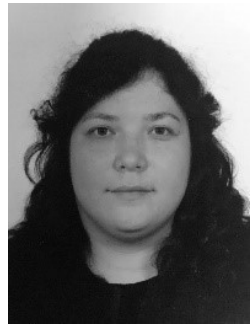

Betul Ilbilgi Yildiz received Bachelor of Science degree in Electrical and Electronic Engineering from Pamukkale University, Denizli, Turkey in 2013 and Master of Science degree in Electrical Engineering in 2017 at University of Texas at Arlington, Texas, USA. She is currently pursuing the Ph.D. degree with the Department of Bioengineering, Imperial College London, London, UK. Her current research interests include analogue and digital systems design.

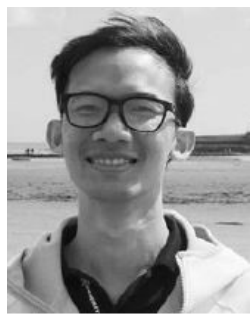

Krit Sujarittam received the B.Eng in mechanical engineering from Chiang Mai University, Chiang Mai, Thailand, in 2016, and the M.Sc. degree in biomedical engineering from Imperial College London, London, U.K., in 2018, where he is currently pursuing the Ph.D. degree in bioengineering with a focus on modeling and interpreting signals generated by microbubbles for therapeutic applications.

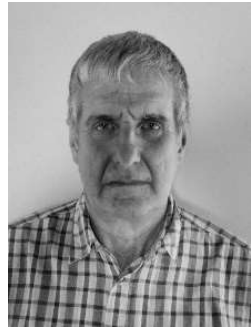

Robert J. Dickinson received the B.A. degree in natural sciences from Cambridge University, Cambridge, U.K., in 1976, and the Ph.D. degree in biophysics from the University of London, London, U.K., in 1980, with a focus on ultrasound signal processing. He has substantial experience in the biocompatibility, electrical safety, and other issues of invasive medical devices, together with commercialization and IP transfer. Dr. Dickinson is a member of the Institute of Physics and Institute of Physics and Engineering in Medicine. $\mathrm{He}$ is a

Chartered Engineer

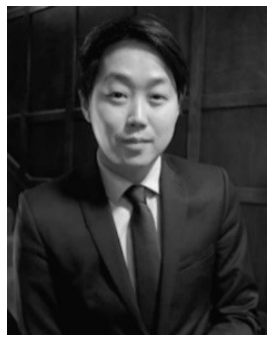

James J. Choi received the B.S.E. degree in computer engineering from The University of Michigan, Ann Arbor, MI, USA, in 2004, and the $\mathrm{Ph} . \mathrm{D}$. degree in biomedical engineering from Columbia University, New York, NY, USA, in 2010. He then conducted his postdoctoral research at the University of Oxford, Oxford, U.K. He is currently a Senior Lecturer with the Department of Bioengineering, Imperial College London, London, U.K. Dr. Choi received the Frederick V. Hunt Postdoctoral Research Fellowship in Acoustics in 2011 and 2012. In 2013, he founded the Noninvasive Surgery \& Biopsy Laboratory, Imperial College London. 